\title{
Factors Influencing the Intended Adoption of Digital Transformation: A South African Case Study
}

\author{
Rion van Dyk \\ Department of Information Systems, \\ University of Cape Town \\ P Bag, Rondebosch, South Africa \\ rionvandyk@gmail.com
}

\author{
Jean-Paul Van Belle \\ CITANDA, Department of Information Systems, \\ University of Cape Town \\ P Bag, Rondebosch, South Africa \\ Jean-Paul.VanBelle@uct.ac.za
}

\begin{abstract}
Organisations worldwide are facing a market pressures which are forcing them to undertake digital transformation projects or initiatives. This research study set out to explore this in more depth, asking questions about the intention of South African (SA) retail organisations to adopt digital transformation and probing into the understanding and perception of digital transformation itself, as well as future intended use cases for available digital technologies. A case study approach was deemed to be an appropriate strategy given the paucity of existing academic research on the topic. Participants in the study had a good understanding of digital transformation and digital technologies, but perceptions were mixed. The most prominent digital transformation initiatives in the $\mathrm{SA}$ retail industry were the adoption of cloud technologies and data analytics. The factors identified in this study, using the Technology, Organisational, and Environmental (TOE) framework, can assist retailers in their decision-making process concerning digital transformation adoption.
\end{abstract}

Key Words: Digital Transformation, Digital Technologies, Strategy, South African Retail Organisations, Technology Adoption, Perceptions, Understanding, TOE, Technology Drivers.

\section{INTRODUCTION}

Many industries have been facing a market shift over the past few years driven by a better response to customer demand which forced enterprises to undertake digital transformation projects or be left behind the competition [1]. Digital transformation refers to a type of strategy that changes an enterprise business model which ultimately provides the customer with variants of tangible product by taking advantage of new or existing digital technologies [2]. The competitive landscape is changing in many industries due to business digitalization. Enterprises face threats of digital disruption from new market entrants while digitally savvy customers are demanding more from the enterprise [3]. Digital transformation affects every enterprise and sector as the market-changing potential of digital technologies is often wider than sales channels, supply chains, products and business processes [4].

One of the biggest challenges enterprises currently face is integrating and exploiting new digital technologies [4].
Digital technologies are tools that enterprises must make use of to get closer to their customers, transform their business processes and empower their employees [5]. Current new digital technologies include cloud computing, mobile, analytics, social media, robotics and Internet of Things (IoT) technologies [6]. These digital technologies can present the enterprise with game-changing opportunities if they are combined with accessibility of enterprise data to enrich their products, services and customer relationships [7].

There is a lack of information around digital transformation, its perceptions and use cases in the SA retail industry to aid its adoption. The main objective of this study was to understand and examine the current perceptions and status of digital transformation within a SA retail organisation. Furthermore, the study aimed to identify factors influencing the intended adoption of digital transformation within the SA retail organisation. This will provide the information and knowledge needed for the retail industry to make informed decisions about the potential future use of digital technologies and how to overcome adoption barriers.

The following propositions are posed to relate the research findings to existing theories and models identified in the literature review.

PR1: There is a lack of understanding of digital transformation in the SA retail industry and perceptions of digital transformation are mixed.

PR2: SA retail industry organisations have identified specific core technologies driving digital transformation.

PR3: The intended adoption of digital transformation by SA retail organisations is affected by specific TOE (Technology, Organisational, \& Environmental) factors.

It is important to study the factors influencing the intended adoption of digital transformation so that enterprises can understand the challenges and address them. Addressing these challenges will be beneficial to the enterprise as it will assist it to create a clear and coherent digital strategy, lead to retaining and attracting top talent, and create a company culture where employees could be innovative and creative. Ultimately, a digital transformed enterprise will be able to easily adapt taking advantage of new opportunities and have a competitive advantage over their competition. 


\section{LITERATURE REVIEW}

\section{A. Digital Transformation}

Digital transformation refers to an enterprise business model that applies new or existing digital technologies and products or services into digital variants to offer a tangible product to their customers [2].

Digital transformation is not only about technology, but it also requires a new way of thinking and strategy by enterprise executives. "Digital transformation is the profound transformation of business and organisational activities, processes, competencies and models to fully leverage the changes and opportunities of a mix of digital technologies and their accelerating impact across society in a strategic and prioritized way, with present and future shifts in mind" [1]. Enterprise digital transformation strategies should include the application of digital technologies to enterprise processes, products and assets to enhance customer value, uncover new monetization opportunities, improve efficiencies and manage risk across the enterprise [8] [9].

\section{B. Digital Technologies}

New digital technologies (social, mobile, analytics, cloud computing and Internet of Things [IoT] technologies) could present the enterprise with game-changing opportunities and existential threats. Leaders in digital transformation apply new digital technologies and related technologies in conjunction with the accessibility of enterprise data to enrich their products, services and customer relationships [7].

\section{Social Media}

The phenomenal and exponential growth of social media and mobile resulted in many organisations realizing that an online presence is required to reach out and connect with their digital savvy customers [10]. Capturing data from tools such as Facebook, LinkedIn and blogs are essential to integrate the information into the sales process [11]. Digital savvy customers follow brands on social media and expect to be able to view store inventory online to enable them to do "showroom" shopping before going into a physical store [10].

\section{Mobility}

Digital technologies have enabled enterprises to make use of mobility and ubiquitous connectivity features providing the enterprise with immediate interaction and access to a wide range of data and computing power and enabling enterprises to analyse their data and make decisions in real time [12]. Mobility resulted that tech-savvy customers across all facets of society completely changed their behaviours, expectations and the way they interact with enterprises [4].

Mobile penetration throughout Africa often thought of as the least digitally populated continent has reached $70 \%$ of its one billion inhabitants. Over $40 \%$ of the world's population has an internet connection. Mobile technology advances allow for the capture of geographical and contextual data that was previously not possible [13]. Digitalization experienced a significant boost with the introduction of smart mobile devices and the applications that run on them [14].
Furthermore, the declining cost of mobile technologies has broadened their potential for worldwide use [13].

\section{Analytics}

Digital technologies provide enterprises with interpretation capabilities, enabling in-depth analysis and exploration of different kinds of data sets. Digital analytical tools coupled with computer-enabled techniques can yield insight to enterprise executives from massive multidimensional datasets enabling them to make use of analytics to make strategic enterprise decisions [13]. About $90 \%$ of the data available today has been produced in the last two years. This data explosion has been driven by new data sources such as digital transactions, mobile devices, embedded sensors and the growing use of social media by the global population. Enterprises could benefit from learning how to capture, absorb, store and analyse their data and turn their data into a valuable asset [14].

Data analytics should be incorporated into new digital products for personalisation reasons, but also to inform other enterprise departments like product development, sales and marketing [15]. Some enterprises are known for their analytical-based approach to ensure they personalise their service and marketing to the need of each of their individual customers by constantly innovating, improving their processes, launching new service based data-driven applications and capabilities [16].

\section{Cloud Computing}

Cloud computing has been defined as "a model for enabling ubiquitous, convenient, on-demand network access to a shared pool of configurable computing resources (e.g., networks, servers, storage, applications, and services) that can be rapidly provisioned and released with minimal management effort or service provider interaction" [17]. Cloud computing technologies enable enterprises to outsource some elements of the IT value chain with benefits for enterprises such as reduced costs, scalability, flexibility, capacity utilisation, higher efficiencies and mobility [18].

\section{Internet of Things (IoT)}

The Internet of things (IoT) refers to a type of network that enables any device to connect to the internet based on stipulated protocols through information sensing equipment to conduct communication and information exchange. The IoT concept has become more practical in recent years due to the exponential growth of the use of smart mobile devices, the growth of data analytics and cloud computing. IoT enables things to be connected at anytime, anywhere, with anything and with anyone ideally using any network, path or service [19]. IoT will force enterprises to digitally transform and will bring fundamental changes to individuals' and society's expectation and perspectives on how technologies and applications work in the world [20]. 


\section{Adoption Barriers}

\section{Digital Strategy}

One of the biggest barriers to digital transformation and digital maturity is the lack of a clear and coherent digital strategy to drive transformation within the enterprise. Mature digital companies realise that digital technologies should be used to achieve strategic enterprise goals [9]. Digital strategy can be defined as "a business strategy, inspired by the capabilities of powerful, readily accessible digital technologies, intent on delivering unique, integrated business capabilities in ways that are responsive to constantly changing market conditions" [7]. Executives should use the enterprise digital strategy to create competitive advantage, value and customer satisfaction by combining existing technology with capabilities of other digital technologies [7].

Talent

Employing, retaining and developing talent within the enterprise is another challenge faced by digital maturing enterprises [9]. Talent was highlighted as one of the top three key influencing factors of successfully implementing digital transformation projects [21]. Having the right talent allows the enterprise to adapt to change and create new opportunities [9]. Digital leaders should embrace the fact that talented individuals are on the lookout for the best digital opportunities and want to work for digitally enabled enterprises [9].

\section{Company Culture}

Digital mature enterprises need to foster a culture where employees are encouraged to take risks, innovate, be creative and create a collaborative work environment [9]. Trying out a lot of things and learning quickly from errors can only be done if a culture of trial-and-error exists within the enterprise [15]. Changing the company culture is a real challenge during digital transformation. Traditional corporate cultures present executives with resistance to change and barriers that need to be overcome to ensure successful digital transformation [22].

\section{Leadership}

A lack of strong top-down leadership that steers digital transformation by setting direction, building momentum and ensuring the enterprise follows through is another barrier to becoming digitally transformed [5]. Enterprise executives are advised to focus on employees, culture, talent, skillset and leadership [12]. Enterprise executives must drive and coordinate digital transformation across the entire enterprise as it touches every functional area. The entire workforce must "digitalize" by fostering an enterprise culture of collaboration between departments whereby employees are enabled to exchange opinions and ideas across departments [15].

\section{IT Function Transformation}

Digital transformation has prompted enterprise IT departments to rethink the architecture platforms within the organisation to ensure new business demands originating from customers and suppliers who require more digital engagement are met [21]. Many IT departments are not set up to be flexible and agile to ensure quick and fast modifications to applications on short notice when required by business departments. Flexibility, agility and the ability to service business requirements on short notice are all trademarks of a digitally transformed enterprise. The role of IT service provider needs to change to a role of consultant, enabler and innovator by applying "new IT concepts" like cross-functional digital teams, enterprise architecture (EA), co-location and IT innovation management [23].

\section{Omni-channel (OC)}

The customer retail experience has been profoundly transformed in the past 10 years by digital transformation projects which integrate digital technologies into the customer shopping experience [24]. Physical stores remain an important part of the customer shopping experience even though mobile device sales and online commerce are accelerating. But the distinction between traditional brick and mortar stores and online sales channels will disappear as the retail industry moves into a new phase, known as Omni-channel (OC) retailing [25]. OC retailing can be defined as the process whereby customers are influenced and move through multiple digital channels in their search and buying process [25].

\section{Technology, Organisation, Environment framework (TOE)}

The TOE framework was proposed by [26]. It "represents how different elements of an organisation (technology, organisation and environment) affect technological innovations and that the framework is suitable for research as it has the flexibility for variance of the factors or measures" [27].

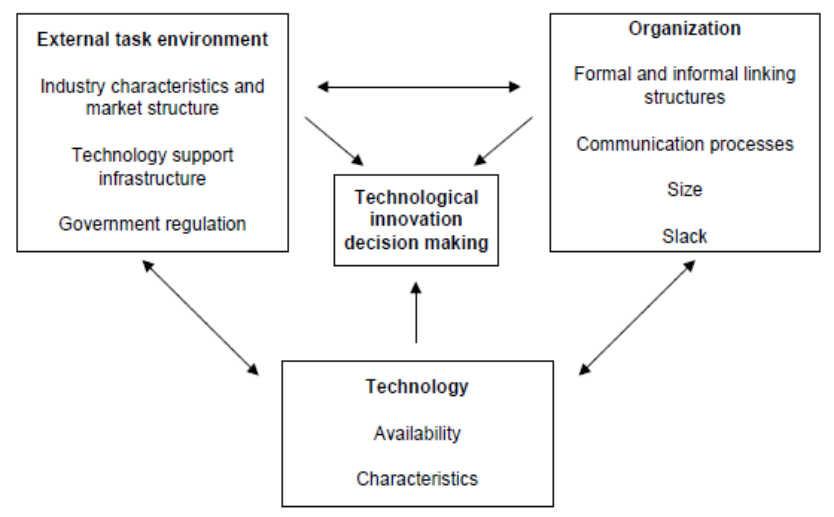

Figure I: The TOE (TeChNOLOGy, Organisation, AND ENVIRONMENT) FRAMEWORK [26]

The TOE technology aspect include all technologies that might be applicable to the enterprise including technologies currently being used by the enterprise, technologies that are available to the enterprise but are not being used and innovative technologies that might enable the enterprise to evolve and adapt [26] [27]. The organisational aspect of the TOE framework relates to all descriptive measures and resources of the enterprise (e.g. the number of employees and communication protocols) which effect may affect executive management decisions with regards to adoption and implementation [26]. Finally, the environmental aspect of the 
TOE framework includes elements, such as the structure of the retail industry in SA, which might affect the adoption of technology within an enterprise [26].

\section{RESEARCH APPROACH AND DESIGN}

The case study approach was chosen as an appropriate strategy to conduct this qualitative study. "A case study examines a phenomenon in its natural setting by employing multiple methods of data collection to gather information from one or a few entities (people, groups, or organisations)" [28]. The researchers actively solicited company documentation before and during interviews which were analysed to support the research.

The research was conducted within a leading African retailer which is part of a retail group with currently more than 4950 stores in 12 African countries. The retailer, used for the case study, currently has 2164 stores across Southern Africa and employs more than 15000 staff. The researchers chose the retailer's head office, in the Western Cape region of SA, as the case site for this study. One of the researchers understands the company culture as he has been employed by the retail group for more than 7 years. Brands within the retail group are well-known household names in Southern Africa. The company has a strong customer focus and their core revenue comes from product sales through their stores to clientele.

The interviewees are listed in table I. All interviewees had degrees or diplomas. The gender split, 9 males and 3 females, is a fair reflection of the demographics found in the retail industry population. The sample population has vast IT retail experience (averaging 24 years) and had seen multiple IT strategies and technologies change over the past two decades.

TABLE I: Research Population \& SAMPLE

\begin{tabular}{|l|l|l|l|}
\hline$\#$ & Position & Gender & Experience \\
\hline P-1 & Team Leader & Male & 24 Years \\
\hline P-2 & Team Leader & Male & 18 Years \\
\hline P-3 & Team Leader & Male & 15 Years \\
\hline P-4 & Team Leader & Male & 21 Years \\
\hline P-5 & DevOps & Male & 10 Years \\
\hline P-6 & Enterprise Architect & Male & 23 Years \\
\hline P-7 & Enterprise Architect & Female & 25 Years \\
\hline P-8 & Director & Male & 29 Years \\
\hline P-9 & Director & Female & 26 Years \\
\hline P-10 & Director & Female & 30 Years \\
\hline P-11 & Director & Male & 32 Years \\
\hline P-12 & CIO & Male & 35 Years \\
\hline
\end{tabular}

The interview protocol consisted of prepared structured (closed-ended) and unstructured (open-ended) questions. The researchers made notes during the interview of the interviewee's comments, personal impressions and observations during the interview while audio recording each interview. The researchers used a thematic approach to analysing the documentation and qualitative data collected during the research using NVivo.

\section{RESEARCH FINDINGS, ANALYSIS \& DISCUSSION}

The following three research questions drove the research:
RQ1: What is the current understanding and perception of Digital Transformation within the SA retail industry?

RQ2: What digital technologies does the SA retail industry perceive are driving implemented to achieve Digital Transformation?

RQ3: What are the factors that influence the intended adoption of Digital Transformation within the organisation?

Twelve interviewees participated in the research strategy involving semi-structured interviews. Theme saturation point occurred from the ninth interview as no new themes emerged in the subsequent three interviews. Therefore, the researchers deemed the sample size sufficient (figure II).

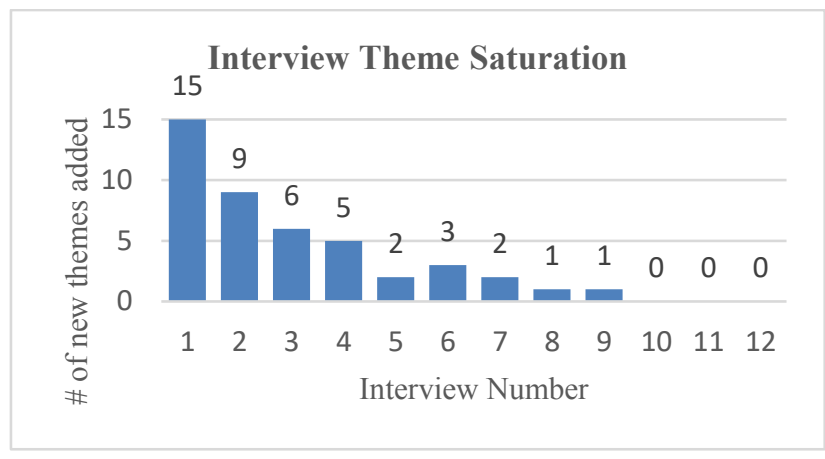

Figure II: INTERVIEW SATURATION PROGRESSION

\section{A. Attitudes towards Digital Transformation}

All interviewees demonstrated a positive attitude toward the adoption of digital transformation, highlighting common technologies that could assist the adoption of digital transformation within the enterprise. The majority of interviewees highlighted common factors that could influence the adoption of digital transformation and mentioned ways to overcome such factors. Furthermore, most interviewees indicated that implementing digital technologies will be beneficial for the enterprise leading to an increase in profit and reducing time to market, ultimately leading to an increase in market share.

\section{B. Technology}

A total of three factors were identified under the technology theme of which the two most significant were "perceived challenges" and "relative advantage", refer to table 3 (the second column shows number of interviewees mentioning the theme). All participants perceived there to be both advantages and challenges by the adoption of digital transformation within the retail industry. The advantages and challenges could have a positive or negative effect on the adoption. The technology theme has been broken down into subthemes and will be discussed further in the next subsections.

Table II: Technology sub-themes (Nr of Mentions)

\begin{tabular}{|l|c|}
\hline Perceived Challenges & $\#$ \\
\hline Infrastructure Impact & 12 \\
\hline Security & 10 \\
\hline Talent / Technical Skills & 4 \\
\hline Relative Advantage & \\
\hline Competitive Advantage & 12 \\
\hline Reduced Cost & 8 \\
\hline
\end{tabular}




\begin{tabular}{|l|c|}
\hline Time to Market & 6 \\
\hline Customer Satisfaction & 6 \\
\hline Available Digital Technologies & \\
\hline G-Suite & 12 \\
\hline Cloud & 12 \\
\hline Artificial Intelligence & 4 \\
\hline Machine Learning & 2 \\
\hline
\end{tabular}

Perceived Challenges (+/-)

A number of adoption barriers were highlighted in the literature review such as the lack of a clear and coherent digital strategy, talent, company culture, IT function transformation and Omni Channel retail capabilities. This was consistent with the responses from the participants with issues around security, workforce talent and resistance to change coming up the most.

It should be highlighted that despite digital strategy being highlighted during the literature review as one of the major challenges affecting the adoption of digital transformation, none of the participants mentioned this as a challenge.

\section{Infrastructure Impact (+)}

Several concerns were raised by participants around how the adoption of digital technologies, specifically cloud technology adoption, would impact their organisation's infrastructure and thus would have a positive impact on digital transformation adoption. Most participants mentioned that the enterprise is currently busy with multiple projects to facilitate cloud adoption and that this will most certainly have a major impact on the existing infrastructure and data centres of the enterprise as P-3 stated "...we are in the process of digital transformation and part of it is moving from our own on premise infrastructure to hosted cloud-based infrastructure". All participants were of the opinion that adopting cloud technologies will result in a saving for the enterprise as maintenance of the on-premise hardware will reduce significantly.

Another infrastructural concern that was raised was the need for high-speed low latency connectivity. P-6: “...with our cloud deployment as our need on high-speed low latency connectivity, for instance, is an infrastructure component that is important to improve on".

\section{Security (-)}

Most participants highlighted that there is a security risk that must be considered with the adoption of cloud technologies as part of digital transformation: "You are moving outside the boundaries of your corporate network so that is a security risk." (P-7)

$\mathrm{P}-7$ is of the opinion that data is an organisation's currency and the organisation should protect that intellectual property (IP) at all cost. P-8 also raised the concern for data protection by stating "....so by making us connected to everything at all times we have to make sure that we've got the bases in place to still protect a corporate organisation like we are". P-2 further solidifies the security concern by stating "You're ultimately putting everything into one place out there in the world, so security really needs to become a top priority".
Further concerns were raised about SA's Protection of Personal Information act (POPI) and payment card information (PCI) compliance. PP-2 states that "...the personal information act POPI requires information to be stored in a certain way with security applied. This actually becomes more important with cloud storage. Also, their payment card information also strict compliance rules that need to be adhered to...". Although enterprises must take regulatory compliance into consideration it's not necessarily a factor that would influence the choice of technology but rather the data generated by the technology as stated by P-11 “...I don't think it necessarily influences the technologies that use but it certainly influences what you do with the data that that technology generates".

\section{Talent - Technical Skills (+/-)}

As mentioned in the literature review, one of the most important critical success factors of digital transformation is to hire new digital talent to compliment or replace the existing workforce to ensure that the enterprise has the "right employees in the right place" [15].

Interviewees gave mixed responses when prompted to comment if the organisation possesses the necessary technical skills to implement digital technologies as part of their digital transformation strategy. Eight participants felt that technical skills within the organisation should not be an adoption barrier in today's fast-changing IT environment: “...it's not technical skills that will stand in our way" [P-8]. P-7 is of the opinion that the organisation could partner with an expert external third party to overcome any technology challenge by stating "We would have to partner with specialized partners in certain areas to take us on the journey. So if we partner with the right people I don't think our technical skills will play such a big role". However, P-2 felt that employees might fear new technologies and that new talent will be required to upskill existing employees by stating "Yes absolutely - the lack of technical skills as far as I can see brings about it a fear. New talent will be required for training of existing employees".

\section{Relative Advantage (+)}

During the data analysis "Relative advantage" emerged as the second biggest factor under the technology major theme and having a positive impact on its adoption. Participants identified a number of perceived benefits (Table III), with customer satisfaction, competitive advantage and reduced cost being cited the most (second column = number of interviewees mentioning the theme).

TABle III: Perceived Benefits of Digital tRANSFORMation

\begin{tabular}{|l|c|}
\hline Customer Satisfaction & 12 \\
\hline Competitive Advantage & 9 \\
\hline Reduced Cost & 7 \\
\hline Time to Market & 6 \\
\hline
\end{tabular}

All participants in the study highlighted that digital transformation would have a positive impact on customer satisfaction, ultimately providing the enterprise with a competitive advantage. Furthermore, by leveraging digital 
technologies enterprises can collect data on customers and use data analysis techniques to offer customers specific goods and services as highlighted by $\mathrm{P}-12$ "...that if you start knowing your customer better you can offer him things that are very specific to him".

\section{Available Digital Technologies (+)}

Available digital technologies emerged as the third biggest factor under the under the technology major theme. An important factor pertaining to technology adoption is the availability of technological innovation [26]. It was important to identify the different digital technologies available as one of the objectives of this study was to identify potential use cases for digital transformation within the SA retail industry.

\section{Google Suite (G Suite) (+)}

G Suite is a set of Google applications that brings together essential services to help your business. This is a hosted service that lets businesses, schools, and institutions use a variety of Google products including Email, Google Docs, and Google Calendar. The adoption of Google Suite (G-Suite) technology, as a replacement of Microsoft Office, by the organisation was highlighted by multiple participants as an important step towards digital transformation. P-2 highlighted the benefit of multiple employees working on the same Google Doc from different locations while having a conference call by stating "I mean it would it's nice to be able to sit and work on a document simultaneously with someone in Durban and Johannesburg while having a conference call".

\section{Cloud (+)}

The majority of participants stated that the use of cloud technologies plays a significant role towards the adoption of digital transformation. "The cloud technology is one of the key technologies to enabled transformation. We are implementing Google cloud platform as our data lake option and we will leverage technologies such as AI in that platform" [P-7]. The importance of providing the retailers and customers with close to real-time information on products and services were highlighted: “... efficiently and close to real-time share information so that our feedback cycle from what we observe in our sales activity in the store can feedback all the way to our planning, manufacture, logistics and merchandising to close that loop so that that becomes more efficient" [P-6].

\section{Artificial Intelligence (AI) (+)}

Artificial Intelligence (AI) refers to the ability of a digital computer, computer-controlled robot or systems to perform tasks commonly associated with intelligent beings like the ability to reason, discover meaning, generalize, or learn from past experience. The organisation will be leveraging their data in the cloud together with AI technologies to generate new valuable insights. P-7 states "Put all of the data together in the cloud and then use the AI technologies to generate new insights for us. I think that there is a huge competitive advantage in machine learning and AI calls that technology will be able to derive insights way faster than a human will possibly be able to on volumes of data".

\section{Machine Learning (MI) (+)}

P-8 explained that the organisation already makes use of machine learning technologies to track if employee's email has been infected by a virus. P-7's personal view is that the organisation's data in the cloud together with machine learning be of great benefit to the enterprise in the future.

\section{Organisation}

A total of nine factors were identified under the organisation theme during the semi-structured interviews. This made it the most populated major theme (Table IV).

TABLE IV: ORGANISATIONAL SUB-THEMES

\begin{tabular}{|l|c|}
\hline Resistance to Change & 12 \\
\hline Financial Resources & 7 \\
\hline Big Data \& Analytics & 7 \\
\hline Digital Technology Readiness & 6 \\
\hline Collaboration & 5 \\
\hline Digital Strategy & 4 \\
\hline Company Culture & 4 \\
\hline Compatibility & 3 \\
\hline Trialability & 3 \\
\hline
\end{tabular}

The high number of factors identified by the participants suggested that organisational factors were dominant in their opinion when considering the adoption of digital transformation within the retail industry. The researchers will discuss these factors in the next subsections.

Resistance to Change (+/-)

The most cited factor under the organisation theme was resistance to change. Most participants highlighted that some employees have been working at the organisation for many years and is used to doing things a certain way: “...we always has employees that are resistant to change so we might be leaving people behind if we don't put in a lot of effort to bring them on board and to take them with us on the journey. One of the factors is that people often say it's always been done in the same way that it's historically resistant to change" [P-8]. $\mathrm{P}-6$ highlighted the fact that before you can address resistance to change you must clearly define the scope of the project by stating “... you need a clear definition of what your digital transformation is". Communication challenges can be addressed by a clear and well-defined scope which is clearly communicated to all employees. P-5 highlighted that as part of addressing the resistance to change within the organisation you may have to address issues with current processes. P-2 mentioned that change management is a very important factor in stating "...change management is such a big thing. If you don't handle it correctly, they won't they won't adopt it, well not easily at least".

\section{Digital Technology Readiness (+)}

Some participants stated that the organisation needs to be prepared to adopt digital technologies. Preparing the organisation for digital technologies so that they are ready and willing to adopt new technologies will have a positive impact. P-7 stated that the organisation currently "... rely heavily on the Gartner hype cycle, specifically the one for emerging technologies and retail technologies" to ensure the 
organisation is kept up to date with technology. Technologies that could present business value are proposed to the retailers to assess the retailer's appetite to adopt the technology. P-1 stated that each new technology is evaluated by a technical forum. The technology is assessed in a "sandbox" environment before testing it as a proof of concept (POC). Technologies are only approved for implementation after a rigorous evaluation process. P-5, who is part of the technical forum, states that each technology is assessed based on the organisation's requirement matrix and "...based on the requirements and our future requirements that we can think of we would select the technology that best fits our area and our vision".

\section{Financial Resources (-)}

Factors relating to financial resources in an organisation such as the cost of adopting new technologies or cost of changing existing technologies have a negative impact on whether an organisation will decide to proceed with implementing technology changes. Return on investment (ROI) plays a major part from the beginning when organisations decide on which technology projects will be implemented: "... if the return on investment is good enough then that's how I would motivate the use of new technology." [P-9]. ROI is very important in retail: "as low cost the retailer we are always striving to find more competitive ways to do things to be more cost-efficient" [P-9]. P-7 highlights the fact in some industries it is more difficult to secure funding for projects that in other by stating "...the retailers are very reluctant. In the banking sector and insurance sector money for major key investments is not such a big issue. In retail, you know the retailers are very reluctant to fork out the chequebook to buy these big investment items that are going to transform the companies".

\section{Big Data \& Analytics (+)}

Data is being generated by a magnitude of sources within the organisation. The organisation harvests as much of the generated data as possible and store it in the cloud to use digital technology to generate new valuable insights for the business. P-7 stated that the organisation "...put[s] all of the data together in the cloud and then use [s] the AI technologies to generate new insights for us". The organisation benefited from the big data and analytics strategy in a number of ways. The organisation was "able to derive insights way faster than a human will possibly be able to on volumes of data" [P-7]. The sharing of information became easier: "...which then makes sharing information processes accessible to staff internally, external parties" [P-6]. Another benefit highlighted was that the adoption of cloud, analytics and the leveraging of big data gave the organisation the ability to better understand and know their customer. Marketing certain products to certain customers improved significantly as more information about the customer where collected, stored and analysed: "...if you can understand your customer you can better fulfil their needs and the only way to do that is with big data and data analysis" [P-2].

\section{Trialability (+)}

"Trialability is the degree to which an innovation may be experimented with on a limited basis..." [29]. A trial or test of the capabilities of digital technologies, in the form of a proof of concept (POC), is a way for an organisation to stifle any doubt or negative perceptions they may have about certain digital technologies and is a positive enabler of adoption. P-6 highlighted the fact that organisations don't always have to be the first to adopt new technologies, especially without proving that the adoption of new technologies will add value: “...you don't need to be the guinea pig and embark on new technology trends without proving that for yourself first. You don't want to go big bang on new stuff, you always want to take baby steps, always have POC's, test it out, monitor if you are achieving your goals and benefits you have set yourself and over time commit to more of that as you see that actually working within the organisation".

\section{Compatibility (+)}

"Compatibility is the degree of how consistent an innovation is perceived to be within an organisation and is affected by internal structures, strategy, values, experience and the needs of the business" [30]. P-7 confirmed that the retail organisation must investigate and demonstrate to the business that new digital technologies will add business value by stating that the organisation must “...determine what business value that is technologies will actually have for our retailers and also the appetite of the retailer to actually adopt that technology".

\section{Company Culture (+/-)}

Company culture can have a positive or negative effect on the adoption of digital transformation within the retail industry: "... if the culture is not ready or your culture is not very open to change, then that could be a barrier for you in terms of digital transformation" [P-7]. Experienced employees might see change as a risk to their careers. It is of utmost importance that the expectation of all employees are managed well, before, during and after embarking on a digital transformation journey as highlighted by P-6 "as people get a bit older, for them, it becomes a risk towards their career where the younger people are more eager to change. I think you need to balance that as well".

\section{Digital Strategy (+/-)}

The lack of a clear and coherent digital transformation strategy driven top-down by top management can be a negative factor adoption factor: “... you need a clear definition of what your digital transformation is. You do need to scope what you mean by that clearly. There is a risk that people might misunderstand what the context is and in terms of that there could be communication challenges" [P-6]. The need for a clear and coherent digital transformation strategy was emphasized by P-5: "...if traditional brick-and-mortar retailers want to survive they will need to transform and grow their e-commerce divisions." Their vision for digital transformation is clearly communicated to the entire organisation and is driven top-down by executives: "I think 
the way the project is approached and communicated and the benefits explained to people will have a big impact on how positive the transformation will be accepted and how successful it will be" [P-9].

\section{Collaboration (+)}

Multiple participants highlighted that collaboration between employees within the organisation will have a positive impact on the adoption of digital transformation. P-8 stated that collaboration must be done between employees and clients to determine the scope for a project by saying "...collaborates with the clients at the highest level. Collaborate with what it is that they're trying to achieve". The need to have communication tools available in an organisation with a distributed workforce was highlighted by P-2: “...we are a large group of people with the distributed management team across $S A$, there's definitely a need for communication tools that bring us together".

\section{Environment}

Only three factors were identified under the environment theme (Table V). The retail customer was by far the most cited factor emphasizing its importance and customer-orientation.

TABLE V: ENVIRONMENTAL SUB-THEMES

\begin{tabular}{|l|c|}
\hline Customer & 12 \\
\hline Competition / Competitive Advantage & 5 \\
\hline Time to Market & 5 \\
\hline Connectivity & 2 \\
\hline
\end{tabular}

\section{Customer (+)}

The retailer customer was highlighted by most as one of the biggest driving factors of digital transformation within the SA retail industry, thus having a positive influence on adoption. The SA retail customer's behaviour when shopping and doing research before and during shopping is changing: "More and more customers are expecting digital transformation with buying online as well as doing online research and just the convenience of shopping anytime anywhere" [P-9]. Customers want to use their smart devices while shopping and expect information on products as and when needed: “...the customer has changed, the customer wants things and information at their fingertips, the customer wants to use new devices and in order to use their device of choice" [P-7].

Retail organisations must adapt and adopt digital technologies to ensure they cater for the needs of the changing customer by enriching the customer experience: [referring to the adoption of digital technologies to enrich the customer's shopping experience]: "...it needs to add customer value, it needs to improve the experience, it needs to reduce cost \& risk, it needs to improve quality" [P-6].

\section{a) Competition / Competitive Advantage (+)}

Organisations have to change, adapt, transform and adopt digital technologies to stay competitive in the SA retail environment: "you will have to adapt and transform to stay competitive" [P-9]. A close eye is kept on what the competition is doing in the market, especially regarding digital technologies, to ensure sales and market share does not decline: “...if our competitor is gaining market share or we are losing sales or whatever because of a competitor employing digital technologies it's definitely something that we will look at" [P-7]. Furthermore, the organisation is more reactive to externally visible digital technology innovations that could affect the customer's shopping behaviour: "When the customer's perception of innovation is positively affected by something, something that's clearly externally visible I do think that forces the organisation to adopt. You need to be more reactive in terms of an external visible technology influence than an internal one" [P-6].

Time to Market (+)

Time to market is a very important factor in a very competitive SA retail market. The perception arose during the semi-structured interview that digital technologies would improve time to market of products, thus having a positive impact on the adoption of digital transformation. Digital transformation coupled with improved business processes and activities will most definitely result in an reduced time to market and cost, increased market share and profit as mentioned by P-2 “...improve our processes and activities if we get this recipe right we will most definitely improve our time to market reduce our costs and increase our market share and hopefully this all leads to increase profits".

\section{Connectivity (-)}

The retail group has a wide footprint of brick and mortar stores throughout Africa requiring an internet connection to trade and be operational. The lack of connectivity throughout Africa was raised as a factor that would negatively impact the adoption of digital transformation when P-3 stated “...I think connectivity is a massive issue given our big footprint and widespread brick-and-mortar stores connectivity is not consistently available everywhere".

\section{E. Technologies Driving Digital Transformation}

The combined analysis from the literature review and semistructured interviews revealed nine core technologies as driving the digital transformation within the SA retail industry. Table VI lists these, ordered by the combined number of participant responses per use case.

TABLE VI: TECHNOLOGIES DRIVING DIGITAL TRANSFORMATION

\begin{tabular}{|l|c|}
\hline e-Commerce Solutions & 12 \\
\hline Big Data \& Analytics & 9 \\
\hline Cloud & 9 \\
\hline Artificial Intelligence & 5 \\
\hline Bots & 4 \\
\hline Machine Learning & 3 \\
\hline Facial Recognition & 3 \\
\hline Self-Checkout & 2 \\
\hline RFID & 2 \\
\hline
\end{tabular}

The most cited technology across the board was to provide the retail group with an e-Commerce solution. All identified technologies were confirmed as relevant to the retail industry.

\section{F. $\quad$ Summary of Findings}

The findings can be used to answer the research questions. 


\section{Understanding and Perception of Digital Transformation}

The research study found that in terms of understanding digital transformation most participants had a general understanding of digital technologies that could be implemented as specific use cases to achieve digital transformation. However, none of the participants indicated that they are aware that digital transformation involves more than implementing certain digital technologies. The researchers found that there are a strong awareness and presence of digital technologies from participant responses received around the use of digital technologies within the SA retail industry which could have a positive or negative influence on adoption. Overall, perceptions from participants were mostly mixed to positive. The mixed perceptions were mostly due to date security concerns surrounding cloud adoption. Thus, the proposition is strongly supported.

\section{Identified Technologies Driving Digital Transformation}

Participants identified nine technologies as driving digital transformation within the retail industry. Cloud technologies and data analytics were the most frequently cited and were the ones which the participants indented implementing. Specific benefits of these technologies included: satisfying changing customer needs, decreasing time to market, increasing customer value, which could be achieved by implementing digital transformation initiatives. Due to the agreement among and the specific technologies and benefits identified, this proposition is strongly supported.

\section{Identified Adoption Barriers}

One of the goals of the study was to identified adoption factors, during the literature review and research strategy, and to provide the knowledge and information needed to the SA retail industry to ensure informed decision are made regarding potential future use. Several positive and negative different factors were identified across the Technology, Organisational and Environmental context.

Perceived challenges were highlighted as the biggest negative factor of the Technological theme, while relative advantage was highlighted as the biggest positive factor in the adoption of digital transformation. Data security when implementing cloud technologies was highlighted as a major concern / negative adoption factor while the adoption of cloud technologies and reduced onsite hardware was seen as a major benefit and stepping-stone to becoming digitally transformed.

The most adoption factors identified was under the Organisational major theme which indicated that participants thought about organisational factors. It became clear that resistance to change would be the biggest organisational barrier to overcome when undertaking digital transformation initiatives. Furthermore, it was highlighted that the cost of implementing digital technologies will play a big role and ultimately return on investment will be a deciding factor.

One of the most important findings that emerged from the Environment major theme was that the retail customer basically drives digital transformation. The changing customer, their needs and the change in how they shop was highlighted by all participants. Thus customer-orientation rather than competitor analysis should drive the adoption of digital transformation in the SA retail sector.

\section{V.CONCLUSION, LIMITATIONS \& FUTURE RESEARCH}

The research study revealed that there was a good understanding of digital transformation from participants within the SA retail industry. However, this could be attributed to their level of training, years of retail experience, the position held within the organisation and the fact that the case site is currently busy with digital transformation projects. Therefore, this could not be generalized across the retail organisation or industry as a whole.

The core technologies perceived by participants as driving digital transformation in the retail industry with e-Commerce solutions, big data \& analytics, and cloud adoption being mentioned the most. The findings from the study also affirmed that there is a consistent association between the adoption of new digital technologies and digital transformation. Finally, the findings identified 22 that affected the adoption of digital transformation within the SA retail sector grouped using the TOE model. Technological factors identified include the imperative to address technical challenges including securing a sound infrastructure (e.g. move some of the infrastructure into the cloud and secure a high-speed, low-latency connection ideally through a locally based cloud vendor); identified security risks include protection of IP as well as customer privacy and hire the correct technical skills. The relative advantages obtained include not only competitive advantage but also reduced costs, reduced time to market and improved customer satisfaction. Enterprises should make extensive use of digital technologies to create a customized personalized user experience through a comprehensive omnichannel approach. Digital technologies enable the enterprise to collect, analyze and interpret data which must be used to optimise their value chain, ultimately increasing profit.

The most prominent organisational issue identified was resistance to change, emphasizing the need for change management and a corporate culture embracing transformation and collaboration. Enterprises that adds specialized digital change agents to their workforce to assist current employees through the digital transformation process will increase their success rate. Assessing the organisation's readiness, possibly through proof of concept sandbox testing is also important - these include aspects of the technology's trialability and organisational compatibility. Digitally mature enterprise must attract and recruit to ensure they don't have skill gaps. Reserving the appropriate financial resources is also important, as is ensuring that big data and effective data analytics are in place is also rated as a crucial step in digital transformation. A clear digital strategy which includes scope and objectives and is driven from the top down rounds off the organisational factors. The crucial environmental factor was, not unsurprising, a focussed customer-orientation for any technologies that are introduced i.e. does it help or add value for the customer as opposed to a knee-jerk competitor-driven 
response. Many of these factors point towards the increased set of IT competencies that retail managers should master. Ultimately, enterprises must analyse the customer's behaviour through their entire shopping experience by making use of big data and analytics. An agile, creative, innovative workforce must elevate the customer's experience resulting in a competitive edge.

The extent to which these findings can be generalized to other countries depends on the structural and environmental similarity of the retail industry with the South African one which operates in a first world/third world context: infrastructure and scarce skills considerations may be more specific, but overall skills, readiness, corporate culture, change management, relative advantage and others are likely to be generalizable to many other country contexts.

Some limitations must be considered when interpreting the results of this study. The study provided a narrow focus on one large retail group, while the retail industry is made up of organisations of varying sizes. The seniority and the position that some of the interviewees fulfil in the case site enterprise resulted that they had limited time available for interviews. Also, the interviewee base was quite small but thematic saturation was reached after the ninth interview.

Future studies around the adoption of digital transformation in the retail sector could be conducted to identify new factors that might impact adoption. The research has identified the need for a study to be conducted across the SA retail industry in order to access a national view on digital transformation in different size retail organisations. In doing so the research will get a more in-depth view of positive and negative factors influencing the indented adoption of digital transformation and more potential use cases could be identified.

\section{VI.REFERENCES}

[1] E. Henriette, M. Feki and I. Boughzala, 2015. The shape of digital transformation: a systematic literature review. MCIS 2015 Proceedings, 431-443.

[2] O. Gassmann, K. Frankenberger and M. Csik. The business model navigator: 55 models that will revolutionise your business. Cambridge: Pearson, 2014. https://doi.org/10.3139/9783446437654.003

[3] S. K. Sia, C. Soh and P. Weill, 2016. How DBS Bank Pursued a Digital Business Strategy. MIS Quarterly, 15(2), 105-121.

[4] L. Hudson and J. Ozanne, 1988. Alternative Ways of Seeking Knowledge in Consumer Research. Journal of Consumer Research, 14(4), 508-521. https://doi.org/10.1086/209132

[5] G. Westerman, D. Bonnet and A. McAfee, 2014. Leading digital: Turning technology into business transformation. Massachusetts: Harvard Business Press, 13-15.

[6] K. Dery, I. M. Sebastian, and N. van der Meulen, "The Digital Workplace is Key to Digital Innovation,” MIS Quarterly, 16(2),2017, 135-152.

[7] I. M. Sebastian, J. W. Ross, C. Beath, M. Mocker, K. G. Moloney and N. O. Fonstad, 2017. How Big Old Companies Navigate Digital Transformation. MIS Quarterly, 197-213.

[8] E. M. Rodgers, 1995. Diffusion of innovations (4th Ed.). New York, NY: Free Press.

[9] G. C. Kane, D. Palmer, A. N. Phillips and D. Kiron, 2017. "Winning the digital war for talent.” MIT Sloan Management Review, 58(2), 17-19.

[10] R. Hansen and S. Sia, 2015. Hummel's Digital Transformation Toward Omni-channel Retailing: Key Lessons Learned. MIS Quarterly, 14(2), $132-149$
[11] D. L. Rogers, 2016. The digital transformation playbook: rethink your business for the digital age, Columbia: Columbia University Press. https://doi.org/10.7312/roge17544

[12] M. H. Ismail, M. Khater and M. Zaki, 2017. Digital Business Transformation and Strategy: What Do We Know So Far? University of Cambridge, Working Paper ITWeb IoT Survey. http://v2.itweb.co.za/index.php?option=com_content\&view=article\&i $\mathrm{d}=166391 \&$ Ite $\mathrm{mid}=3087$, [15 April 2017]

[13] D. L. Soule, N. Carrier, D. Bonnet and G. F. Westerman, 2014. Organizing for a Digital Future: Opportunities and Challenges. MIT Center for Digital Business and Capgemini Consulting. Working Paper. https://doi.org/10.2139/ssrn.2698379

[14] H. Gimpel, S. Hosseini, R. Huber, L Probst, M. Röglinger and U. Faisst, Structuring Digital Transformation: A Framework of Action Fields and its Application at ZEISS. Journal of Information Technology Theory and Application, 19(1), 2018, 32-54.

[15] T. Hess, C. Matt, A. Benlian and F. Wiesböck, 2016. Options for Formulating a Digital Transformation Strategy. MIS Quarterly, 15(2), 123-139.

[16] H. Ghasemkhani, D. L. Soule and G. F. Westerman, Competitive Advantage in a Digital World: Toward an Information-Based View of the Firm. MIT Center of Digital Business, Working Paper, (2014). https://doi.org/10.2139/ssrn.2698775

[17] M. Lane, A. Shrestha and O. Ali, 2017. Managing the risks of data security and privacy in the cloud: a shared responsibility between the cloud service provider and the client organisation. Seoul, The Bright Internet Global Summit.

[18] M. Carroll, A. Van Der Merwe and P. Kotze, Secure cloud computing: Benefits, risks and controls. Information Security South Africa (ISSA), IEEE. 2011, pp. 1-9. https://doi.org/10.1109/ISSA.2011.6027519

[19] K. K. Patel, S. M. Patel and P.S.A Professor, 2016. Internet of ThingsIOT: definition, characteristics, architecture, enabling technologies, application \& future challenges. International Journal of Engineering Science and Computing, 6(5).

[20] O. Vermesan and J. Bacquet, 2017. Cognitive Hyperconnected Digital Transformation: Internet of Things Intelligence Evolution, Eds. Demark: River Publishers. https://doi.org/10.13052/rp-9788793609105

[21] O. A. Sawy, P. Kræmmergaard, H. Amsinck and A. L. Vinther, 2015. How LEGO Built the Foundations and Enterprise Capabilities for Digital Leadership. MIS Quarterly, 15(2).

[22] A. Singh and T. Hess, 2017. How Chief Digital Officers Promote the Digital Transformation of their Companies. MIS Quarterly, 16(1).

[23] N. Urbach, P. Drews, and J. Ross, 2017. Digital business transformation and the changing role of the IT Function. comments on the special issue. MIS Quarterly, 16(2).

[24] E. Huré, K. Picot-Coupey and C. L. Ackermann, 2017. Understanding Omni-channel shopping value: A mixed-method study. Journal of Retailing and Consumer Services, 39, 314-330. https://doi.org/10.1016/j.jretconser.2017.08.011

[25] P. C. Verhoef, P. K. Kannan and J. J. Inman, 2015. From multi-channe retailing to Omni- channel retailing: introduction to the special issue on multi-channel retailing. Journal of retailing, 91(2), 174-181. https://doi.org/10.1016/i.jretai.2015.02.005

[26] R. DePietro, E. Wiarda, and M. Fleischer, Processes of Technological Innovation. Massachusetts, MA: Lexington Books, 1990.

[27] J. Baker, The technology-organization-environment framework Information systems theory, New York, NY: Springer, 2012, pp. 231 245. https://doi.org/10.1007/978-1-4419-6108-2 12

[28] I. Benbasat, D. K. Goldstein and M. Mead, "The case research strategy in studies of information systems," MIS Quarterly, 1987, 369-386. https://doi.org/10.2307/248684

[29] M. Rodriguez, R. M. Peterson and H. Ajjan, 2015. CRM/social media technology: impact on customer orientation process and organizational sales performance. Ideas in Marketing: Finding the New and Polishing the Old, 636-638. Springer: Cham. https://doi.org/10.1007/978-3-31910951-0 233

[30] A. Lin and N. C. Chen, 2012. Cloud computing as an innonvation: Perception, attitude, and adoption. International Journal of Information $\begin{array}{lll}\text { Management, 32(6), 533-540. } & \text { 320 }\end{array}$ https://doi.org/10.1016/j.ijinfomgt.2012.04.001 